\section{Development of Plasma Vitellogenin Assay for Estrogenic Effects of Endocrine-Disrupting Chemicals Using Ovariectomized Goldfish (Carassius auratus)}

\author{
Hiroshi Ishibashi, ${ }^{a, c}$ Makito Kobayashi, ${ }^{b}$ \\ Yuki Tomiyasu, ${ }^{a}$ Maki Miyahara, ${ }^{a}$ \\ Katsuyasu Tachibana, ${ }^{c}$ \\ Mutsuyosi Tsuchimoto, ${ }^{c}$ \\ and Koji Arizono $*, a$
}

${ }^{a}$ Faculty of Environmental and Symbiotic Sciences, Prefectural University of Kumamoto, 3-1-100 Tsukide, Kumamoto 8628502, Japan, ${ }^{b}$ Department of Biology, Division of Natural Sciences, International Christian University, 3-10-2 Osawa, Mitaka, Tokyo 181-8585, Japan, and ' Graduate School of Science and Technology, Nagasaki University, 1-14 Bunkyo-machi, Nagasaki 852-8521, Japan

(Received November 12, 2003; Accepted November 18, 2003; Published online November 26, 2003)

Plasma vitellogenin (VTG) assay was developed using ovariectomized goldfish (Carassius auratus) for determining the estrogenic effects of endocrine-disrupting chemicals. In a laboratory study, we assessed the estrogenic activity of commercial fish diets by using a diet for ornamental carp (CD) and a casein-based formulated fish diet (FD), which was shown to not contain soybean or fish meal in a previous study. In ovariectomized fish, plasma VTG concentrations were significantly higher in the CD-fed group than in the FDfed group. These results indicate that the estrogen activity of CD may be high enough to cause induction of plasma VTG in ovariectomized goldfish as previously observed in male goldfish. Moreover, the effect of estrogen on plasma VTG induction was confirmed by significant plasma VTG production following the exposure of FD-fed ovariectomized goldfish to a nominal estradiol-17 $\beta$ concentration of $100 \mu \mathrm{g} / \mathrm{l}$ for 31 days. Our data suggest that induction of plasma VTG using ovariectomized goldfish is a good tool for evaluating

\footnotetext{
*To whom correspondence should be addressed: Faculty of Environmental and Symbiotic Sciences, Prefectural University of Kumamoto, 3-1-100 Tsukide, Kumamoto 862-8502, Japan. Tel.: +81-96-383-2929 (ext. 486); Fax: +81-96-383-6062; E-mail: arizono@pu-kumamoto.ac.jp
}

the estrogenic effects of endocrine-disrupting chemicals.

Key words — ovariectomized goldfish, vitellogenin, estrogenic effect, phytoestrogen

\section{INTRODUCTION}

Various endocrine-disrupting chemicals (EDCs) are known to impact the development and function of endocrine systems in animals and humans. ${ }^{1,2}$ Observations of sexual abnormalities in wild fish due to exposure to environmental pollutants have raised widespread concern about some aquatic environmental EDCs, such as estrogens and xenoestrogens. Many EDCs may also cause adverse effects in freshwater and marine water fish populations. ${ }^{3,4)}$ Soybeans contain estrogenic isoflavones and derivatives such as coumestrol, formononetin, daidzein, biochanin A, genistein and equol, which can also disturb reproductive function in mammals. ${ }^{5,6)}$ These isoflavonic compounds may act as estrogen agonists by binding to estrogen receptors on target tissues and enhancing RNA synthesis ${ }^{7,8)}$ or acting antagonistically to block any RNA replication when bound to estrogen receptors thereby producing an antiestrogen physiological effect. ${ }^{9)}$ In our previous study, we demonstrated significant increased plasma vitellogenin (VTG, egg yolk protein precursor) in male goldfish fed the phytoestrogenenriched fish diet (FD) ${ }^{10)}$

Recently, various screening and testing systems for EDCs in fish have been established through cooperation of the Organization for Economic Cooperation and Development (OECD) and U.S. Environmental Protection Agency (EPA). ${ }^{11,12)}$ Model test organisms include advanced fish strains such as fathead minnow (Pimephales promelas), zebrafish (Danio rerio) and Japanese medaka (Oryzias latipes). However, goldfish (Carassius auratus), a cyprinid species, would be suitable for the evaluation of EDCs. Goldfish are used intensively for investigating the reproductive endocrinology of fish and are easily handled and maintained. Therefore, in the recent study, the monitoring of plasma VTG levels in male goldfish has been used to assess estrogenic effects. ${ }^{13,14)}$ However, it is difficult to obtain male goldfish throughout the year due to the difficulty in measuring the appearance of secondary sex characteristics except at sexual maturity. More- 
over, male fish is known to produces small amount of estrogen. ${ }^{15)}$

In this study, a plasma VTG assay for estrogenic effects of EDCs was developed using ovariectomized goldfish, which can be supplied throughout the year. We assessed the estrogenic activity of the phytoestrogen-enriched commercial fish diets as a biomarker of estrogenic effect using plasma VTG induction in ovariectomized goldfish which does not produce endogenous gonadal estrogens. We also investigated the response for estrogen on plasma VTG induction in ovariectomized goldfish, and discussed the utility of ovariectomy.

\section{MATERIALS AND METHODS}

Fish and Exposure Conditions — Mature twoyear-old female goldfish (Carassius auratus) weighting 13.1-28.1 $\mathrm{g}$ were obtained from a local distributor. Ovariectomy was conducted as described by Kobayashi and Stacey, ${ }^{16)}$ with a postoperative survival rate of over $90 \%$. These ovariectomized, shamoperated, and unoperated female goldfish were not fed for a month before the start of the experiment, and were kept in a 25-1 glass tank under natural conditions, as in the previous study. ${ }^{10)}$ Fish diets used in this study were a diet for ornamental carp (CD) and a newly developed casein-based formulated diet "No. 2," which does not contain soybean or fish meal. In our previous study, the phytoestrogen content of CD was shown to be much higher than that in FD, and fish fed the CD diet had significantly higher VTG levels than the fish fed FD based on the plasma VTG assay of male goldfish. ${ }^{10)}$

Ovariectomized (6 fish, 2 groups), unoperated goldfish (4 fish, 2 groups), and sham-operated (4 fish, 1 groups) were fed $1.0 \%$ body weight volume of one of the two diets, FD or CD every 2 days for 31 days between May and June 2000. During the experimental period, the fish were maintained in dechlorinated tap water in 25-1 glass tanks at 23$26^{\circ} \mathrm{C}$ under a $12 \mathrm{hr}$ light- $12 \mathrm{hr}$ dark photoperiod. A portion of the tank water was exchanged with dechlorinated tap water every $72 \mathrm{hr}$.

To confirm the response for estrogen in ovariectomized goldfish, experimental fish was exposed to the nominal concentrations of $100 \mu \mathrm{g} / \mathrm{l}$ estradiol- $17 \beta$ (E2, Sigma Chemical Industries Ltd., Tokyo, Japan) dissolved in dechlorinated tap water at $23-26^{\circ} \mathrm{C}$ for 31 days. Control fish were exposed to the solvent carrier only $(0.1 \mathrm{ml} / \mathrm{l}$ dimethyl sulfoxide). Each group of fish was kept in a 25-1 glass tank maintained under $12 \mathrm{hr}$ light-12 hr dark photoperiod. During exposure periods, water in the tanks was changed every $24 \mathrm{hr}$. The fish were fed $1.0 \%$ body weight volume of FD every 2 days for 31 days.

There is no institutional animal care and use committee for the fish in Japan. However, for the protection of animals, the number of fish used in this study was kept to a minimum.

Sample Collection — At the end of the experiment, the body length and body weight were measured and blood samples were taken from the caudal vasculature with a heparinized syringe and needle. Blood samples were transferred into a centrifuge tube and mixed with a $0.1 \%$ volume of saline containing $10000 \mathrm{KIU} / \mathrm{ml}$ aprotinin, $0.1 \%$ phenylmethylsufonyl fluoride, and $14.0 \mathrm{U} / \mathrm{ml}$ heparin. Blood was centrifuged at $3000 \mathrm{rpm}$ for $20 \mathrm{~min}$, and the plasma was stored at $-30^{\circ} \mathrm{C}$ until assayed. All preparative procedures were carried out at $4^{\circ} \mathrm{C}$. The body, ovaries and hepatopancreas were weighed, and gonadosomatic (GSI, gonad weight $\times 100 /$ body weight) and hepatosomatic index (HSI, hepatopancreas weight $\times 100 /$ body weight) were calculated, respectively.

Measurement of Plasma VTG — Concentrations of VTG in blood plasma were determined by enzyme-linked immunosorbent assay (ELISA) as described by Ishibashi et al. ${ }^{17)}$ Purified goldfish VTG $(7.8,15.6,31.2,62.5,125,250$ and $500 \mathrm{ng} / \mathrm{ml})$ was used to construct a standard curve, and VTG in diluted samples was measured in duplicate. The assays were performed at room temperature. Concentrations of VTG in blood plasma samples were calculated from the linear part of the log-transformed goldfish VTG standard curve. The detection limit of VTG in the present study was $0.040 \mu \mathrm{g} / \mathrm{ml}$.

\section{RESULTS}

\section{Growth, GSI, HSI and Plasma VTG Levels in Ovariectomized, Sham-Operated and Unoperated Fmale Goldfish Fed Commercial Fish Diets}

There were no significant differences in the body lengths and body weights among all treatment groups of fish fed the FD or CD diet for 31 days (data not shown). There was no significant difference in GSI among the FD- and CD-fed ovariectomized goldfish (Fig. 1A). On the other hand, there were no sig- 


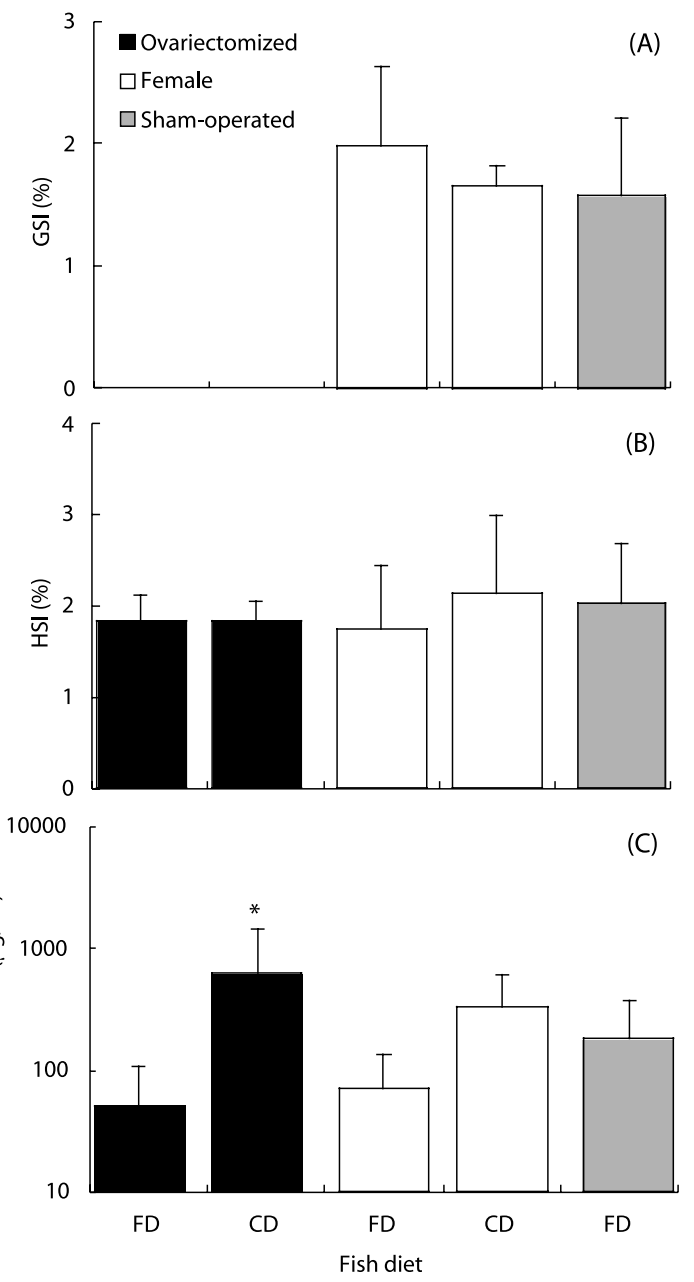

Fig. 1. Levels of GSI (A), HSI (B) and Plasma VTG (C) of Ovariectomized, Control and Sham-Operated Female Goldfish, which were Fed Two Diets (FD and CD) for 31 days

Each group of fish was fed $1.0 \%$ body weight volume of FD or CD every 2 days for 31 days. Values shown are the mean GSI, HSI and VTG concentrations. Error bars represent the standard deviation of the mean. *Significantly different when compared to the FD-fed ovariectomized fish ( $p<0.05$, Mann-Whitney U test with Bonferroni's adjustment).

nificant differences in HSI among all treatment groups (Fig. 1B). Plasma VTG concentrations in ovariectomized fish (Fig. 1C) were significantly higher in the CD-fed group $(622.53 \pm 827.59 \mu \mathrm{g} / \mathrm{ml})$ than in the FD-fed group $(51.29 \pm 56.40 \mu \mathrm{g} / \mathrm{ml})$ by the Mann-Whitney U test with Bonferroni's adjustment $(p<0.05)$. On the other hand, VTG in female fish was higher in the CD-fed group (329.08 \pm $269.82 \mu \mathrm{g} / \mathrm{ml})$ than in the FD-fed group $(70.49 \pm$ $64.23 \mu \mathrm{g} / \mathrm{ml}$ ), but the differences were not statistically significant. In the sham-operated fish fed the FD diet, the plasma concentration of VTG was $180.66 \pm 197.80 \mu \mathrm{g} / \mathrm{ml}$.

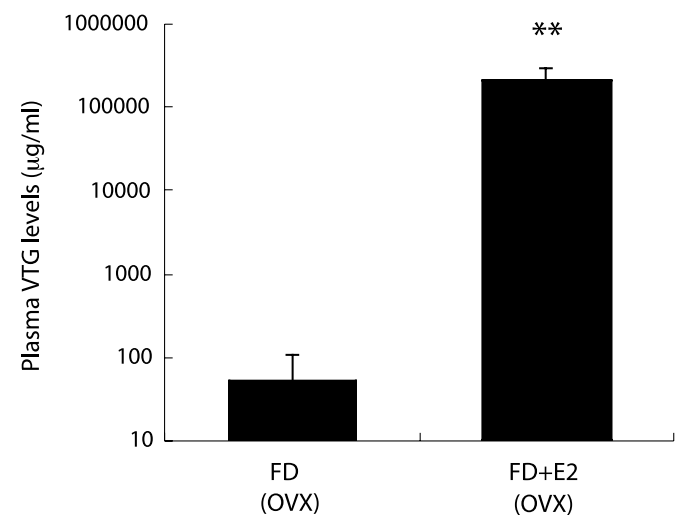

Fig. 2. Plasma VTG Concentrations of FD-Fed Ovariectomized Goldfish after Exposure to $100 \mu \mathrm{g} / \mathrm{l}$ E2 for 31 days

Values are shown as the mean VTG concentration. Error bars represent the standard deviation of the mean. $* *$ Significantly different when compared to the FD-fed control group fish $(p<0.01$, MannWhitney $\mathrm{U}$ test). OVX = ovariectomized goldfish.

\section{Growth, GSI, HSI and Plasma VTG Levels in FD- Fed Ovariectomized Goldfish Exposed to Estra- diol-17 $\beta$}

In ovariectomized fish that were fed the FD diet and exposed or not exposed (control) to E2 for 31 days, there were no significant differences in the level of body length and body weight of both groups (data not shown). There were also no significant differences in GSI (control, 0\%; E2 treatment group, $0 \%$ ) and HSI (control, $1.85 \pm 0.27 \%$; E2 treatment group, $1.99 \pm 0.45 \%$ ) values. In FD-fed ovariectomized goldfish, plasma VTG levels were significantly higher in the group exposed to E2 at $100 \mu \mathrm{g} /$ 1 for 31 days compared to the control group $(p<0.01$, Mann-Whitney U test) (Fig. 2).

\section{DISCUSSION}

In this study, plasma VTG assay was developed for testing the estrogenic effect of EDCs using ovariectomized goldfish. The postoperative survival rate of ovariectomy was over $90 \%$. All fish had a good health status during the experimental period. The high survival rate of ovariectomized goldfish was important for conducting this screening test for the estimation of estrogenic effects on ovariectomized goldfish. The ovariectomized goldfish fed the FD diet were exposed to the nominal E2 concentration of $100 \mu \mathrm{g} / \mathrm{l}$ for 31 days to confirm the response of estrogen on induction of plasma VTG. As a result, significant responses in plasma VTG production were observed with E2. Although further studies are 
required to confirm the response for low-dose estrogen on ovariectomized goldfish, these results suggest that induction of plasma VTG using ovariectomized goldfish is a good tool for the evaluation of estrogenic effects of EDCs as well as the results of previous studies using male goldfish. ${ }^{17)}$

Estrogenic effects of soybeans have been widely described in many animals. ${ }^{18,19)}$ First, we assessed the estrogenic activity of fish diets for biomarkers of estrogenic effects using plasma VTG synthesis in ovariectomized goldfish. Ovariectomized, shamoperated, and unoperated female goldfish were fed two diets, (FD and CD) for 31 days. Plasma VTG levels were significantly higher for CD-fed ovariectomized goldfish compared to the FD-fed ovariectomized fish. In our previous study, we also demonstrated significantly increased plasma VTG in male goldfish fed a phytoestrogen-enriched fish diet, such as CD. ${ }^{10)}$ Estrogenic effects observed in ovariectomized goldfish were similar to those found in our previous paper. Therefore, the progressive increase in plasma VTG of ovariectomized goldfish when fed a $1 \%$ per body weight ration every 2 days indicates the presence of phytoestrogens in $\mathrm{CD}$.

Pelissero et al. ${ }^{20)}$ reported that the estrogenic activity of genistein, daidzein, equol and coumestrol were biologically evaluated by production of VTG in yearling Siberian sturgeon (Acipenser baeri) and compared to the activity resulting from exposure to E2. Genistein, daidzein, equol and coumestrol all had estrogenic activity as assessed by their induction of hepatic synthesis of VTG, and coumestrol seemed to be the most potent compound. We evaluated the estrogenic activity of diets using the yeast two-hybrid assay and the total content of genistein and daidzein to study the relationship between estrogenic activity and the phytoestrogen content. ${ }^{21)}$ Our results indicated that the genistein content contributes to the estrogenic activity of the diets. In the previous study, the effect on VTG production when fed a commercial fish diet was not evaluated, and we believe that this must be addressed because not only are there variable responses to fish diets but also in the normal physiological VTG concentration before an experiment to assess EDCs. However, it is possible that estrogenic substances in commercial fish diets may interact with test chemical compounds by binding to the estrogen receptor and the possibility that these estrogenic effects may be masked in the screening and testing programs can not be discounted. Phytoestrogens such as coumestrol and equol have higher binding affinity for the estrogen receptor than for the synthetic chemicals known to have estrogenic activity, such as bisphenol A and nonylphenol. ${ }^{22)}$ Our results suggest that there is a possible difficulty in evaluating the estrogenic effects of EDCs by in vivo screening and testing systems using plasma VTG production in fish as a biomarker because the fish diet might include high concentrations of phytoestrogens, which also act as EDCs.

In summary, plasma VTG assay for estrogenic effects of EDCs was developed using ovariectomized goldfish which does not produce endogenous gonadal estrogens. Significant responses in plasma VTG production in ovariectomized goldfish were observed following 31-day exposure to $100 \mu \mathrm{g} / \mathrm{l} \mathrm{E} 2$. We also assessed the estrogenic effects of commercial fish diets tested in the previous study. These results indicate that the phytoestrogens, such as genistein, in CD could cause the production of plasma VTG in ovariectomized goldfish. Therefore, we recommend the use of a standardized, open-formula diet in which estrogenic substances have been reduced to levels that will not alter the results of studies that are influenced by exogenous estrogens. Our data suggest that plasma VTG production in ovariectomized goldfish is a good tool for the evaluation of estrogenic effects of EDCs.

\section{REFERENCES}

1) Colborn, T., vom Saal, F. S. and Soto, A. M. (1993) Developmental effects of endocrine-disrupting chemicals in wildlife and humans. Environ. Health Perspect., 101, 378-384.

2) Colborn, T., Dumanoshi, D. and Myers, J. P. (1996) Our Stolen Future, Dutton, NY, U.S.A.

3) Knudsen, F. R., Schou, A. E., Wiborg, M. L., Mona, E., Tollefsen, K., Stenersen, J. and Sumpter, J. P. (1997) Increase of plasma vitellogenin concentration in rainbow trout (Oncorhynchus mykiss) exposed to effluents from oil refinery treatment works and municipal sewage. Bull. Environ. Contam. Toxicol., 59, 802-806.

4) Munkittrick, K. R., McMaster, M. E., McCarthy, L. H., Servos, M. R. and Van Der Kraak, G. J. (1998) An overview of recent studies on the potential of pulp-mill effluents to alter reproductive parameters in fish. J. Toxicol. Environ. Health. B Crit. Rev., 1, 347-371.

5) Adams, N. R. and Sanders, M. R. (1988) Persistent infertility in ewes after prolonged exposure to oestradiol-17ß. J. Reprod. Fertil., 84, 373-378. 
6) Obst, J. M. and Seamark, R. F. (1972) Plasma hormone levels during pregnancy and parturition in ewes grazing Yarloop clover patures. J. Reprod. Fertil., 29, 146-147.

7) Shutt, D. A. and Cox, R. I. (1972) Steroid and phytooestrogen binding to sheep uterine receptors in vitro. J. Endocrinol., 52, 299-310.

8) Thompson, M. A., Lasley, B. L., Rideout, B. A. and Kasman, L. H. (1984) Characterization of the estrogenic properties of a nonsteroidal estrogen, equol, extracted from urine of pregnant macaques. Biol. Reprod., 31, 705-713.

9) Adlercreutz, H., Hockerstedt, K., Bannwart, C., Bloigu, S., Hamalainen, E., Fotsis, T. and Ollus, A. (1987) Effect of dietary components, including lignans and phytoestrogens, on enterohepatic circulation and liver metabolism of estrogens and on sex hormone binding globulin (SHBG). J. Steroid Biochem., 27, 1135-1144.

10) Ishibashi, H., Kobayashi, M., Koshiishi, T., Moriwaki, T., Tachibana, K., Tsuchimoto, M., Soyano, K., Iguchi, T., Mori, C. and Arizono, K. (2002) Induction of plasma vitellogenin synthesis by the commercial fish diets in male goldfish (Carassius auratus) and dietary phytoestrogens. $J$. Health Sci., 48, 427-434.

11) Organization for Economic Cooperation and Development (1999) Final report of the fish expert consultation meeting, London, U.K., October 2829, Environmental Health and Safety Division, Paris, France.

12) U.S. Environmental Protection Agency (1998) Endocrine Disruptor Screening Program: Proposed Statement of Policy. Fed. Regist., 248, 7154271568.

13) Hirai, M., Oda, N., Fujino, H., Fukada, H. and Hara, A. (2003) Development of ELISA and SRID for carp vitellogenin and application to the exposure examination using goldfish. In The 5th Annual Meeting of Japan Society of Endocrine Disrupters Research, p. 237.

14) Taharu, R., Sugihara, K., Tanaka, M., Ohashi, K., Kitamura, S., Ohta, S. and Hara, A. (2003) Metabo- lism of endocrine disrupters in fish and the influence on the action. In The 5th Annual Meeting of Japan Society of Endocrine Disrupters Research, p. 322.

15) Matsumoto, T., Kobayashi, M., Nihei, Y., Kaneko, T., Fukada, H., Hirano, K., Hara, A. and Watabe, S. (2002) Plasma vitellogenin levels in male common carp Cyprinus carpio and crucian carp Carassius cuvieri of lake Kasumigaura. Fisheries Sci., 68, 1053-1064.

16) Kobayashi, M. and Stacey, N. E. (1990) Effects of ovariectomy and steroid hormone implantation on serum gonadotropin levels in female goldfish. Zool. Sci., 7, 715-721.

17) Ishibashi, H., Tachibana, K., Tsuchimoto, M., Soyano, K., Ishibashi, Y., Nagae, M., Kohra, S., Takao, Y., Tominaga, N. and Arizono, K. (2001) In vivo testing system for determining the estrogenic activity of endocrine-disrupting chemicals (EDCs) in goldfish (Carassius auratus). J. Health Sci., 47, 213-218.

18) Axelson, M., Sjovall, J., Gustafsson, B. E. and Setchell, K. D. (1984) Soya a dietary source of the non-steroidal oestrogen equol in man and animals. J. Endocrinol., 102, 49-56.

19) Pelissero, C. and Sumpter, J. P. (1992) Steroid and "steroid-like" substances in fish diets. Aquaculture, 107, 283-301.

20) Pelissero, C., Cuisset, B. and LeMenn, F. (1989) The influence of sex steroids in commercial meals and fish diets on plasma concentration of estrogens and vitellogenin in cultured Siberian sturgeon Acipense baeri. Aquat. Living Resour., 2, 161-168.

21) Miyahara, M., Ishibashi, H., Inudo, M., Nishijima, H., Iguchi, T., Guillette, L. J., Jr. and Arizono, K. (2003) Estrogenic activity of a diet to estrogen receptors $-\alpha$ and $-\beta$ in an experimental animal. $J$. Health Sci., 49, 481-491.

22) Nishihara, T., Nishikawa, J., Kanayama, T., Dakeyama, F., Saito, K., Imagawa, M., Takatori, S., Kitagawa, Y., Hori, S. and Utsumi, H. (2000) Estrogenic activities of 517 chemicals by yeast twohybrid assay. J. Health Sci., 46, 282-298. 